\title{
Factores que interfieren en el incumplimiento de las expectativas reproductivas de las mujeres españolas
}

\author{
Factors that interfer in breach of the reproductive \\ expectations of spanish women
}

Aidée Baranda Ortiz • aidee_baranda001@ehu.eus

UNIVERSIDAD DEL PAÍS VASCO/EUSKAL HERRIKO UNIBERTSITATEA, BECARIA DEL DEPARTAMENTO DE SOCIOLOGÍA Y TRABAJO SOCIAL

Recibido: 04/11/2019

Aceptado: 26/11/2019

\section{Resumen}

El objetivo de este artículo consiste en comprobar la brecha entre el número de hijos deseados y el número de hijos reales en las mujeres españolas y conocer los motivos personales y los factores estructurales que pueden incidir en dicha brecha. Para ello, se han utilizado los datos de la Encuesta de Fecundidad 2018 del Instituto Nacional de estadística. En concreto, se han seleccionado a mujeres entre 45 y 55 años, que son las que ya han finalizado su ciclo reproductivo y se han realizado dos modelos de regresión logística binaria, uno para las mujeres que no han tenido hijos, y otro para aquellas que han sido madres, pero han tenido menos de los deseados. Los resultados apuntan a que dicha brecha existe y que el ideal de los dos hijos sigue arraigado. También se observa que un retraso en la edad de la primera maternidad hace que el número final de hijos sea menor que el deseado. Este grupo de mujeres también menciona los problemas de salud, la falta de recursos económicos y a la dificultad de compatibilizar la vida laboral y familiar como motivos por los que no han cumplido sus deseos reproductivos. La infecundidad se da principalmente como consecuencia de una decisión personal de no querer ser madre y por problemas de salud. Respecto al nivel de estudios, se ve que aquellas con estudios primarios, en comparación a las mujeres con estudios universitarios, son las que desearían haber tenido hijos.

Palabras clave: Fecundidad; Infecundidad; Número de hijos deseados; Número de hijos reales; España.

\begin{abstract}
The objective of this article is to verify the gap between the desired number of children and the real number of children in Spanish women and to know the personal reasons and the structural factors that can influence this gap. Data have been used from the 2018 Fertility Survey of the National Statistics Institute. Specifically, women between 45 and 55 years of age have been selected, who are those who have already completed their reproductive cycle and two binary logistic regression models have been made, one for women who have not had children, and another for those who have been mothers, but they have had less than desired. The results suggest that this gap exists and that the ideal of the two children is still rooted. It is also observed that a delay in the age of first maternity causes the final number of children to be less than desired. This group of women also mentions health problems, lack of financial resources and the difficulty of making work and family life compatible as reasons why they have not fulfilled their reproductive desires. Infertility occurs mainly as a result of a personal decision not to want to be a mother and due to health problems. Regarding the level of education, it is seen that those with primary studies, compared to women with university studies, are those who wish they had children.
\end{abstract}

Keywords: Fertility; Childlessness; Desired number of children; Real number of Children; Spain. 


\section{INTRODUCCIÓN}

En el año 1998, España registró el mínimo histórico en niveles de fecundidad, 1,15 hijos por mujer, después de que cuando acabase la dictadura se situase en 2,8, muy por encima de la media europea $(2,1)$. A partir de ese año, el Índice Sintético de Fecundidad (ISF) seguido manteniendo por debajo del nivel de reemplazo generacional, 2,1 hijos por mujer necesarios para que la población se mantenga. Desde entonces y encabezado por España, se acuñó el término lowest-low-fertility para aquellos países que tuviesen una tasa de fertilidad total por debajo de 1,3 hijos por mujer (Kohler, Billari, \& Ortega, 2004).

Sin embargo, a principios de siglo, se produjo una leve tendencia hacia la recuperación llegando en 2008 a 1,45 hijos por mujer, pero esta se vio interrumpida por la crisis económica (Martín Patino, 2017), volviendo a disminuir el ISF. Actualmente el ISF es de 1,31, y aunque la mayoría de los países desarrollados se encuentran por debajo del mismo, España tiene uno de los más bajos del mundo. El hecho de que cada vez las mujeres retrasen la maternidad y que aumente el porcentaje de mujeres que no tienen hijos (childnessless ${ }^{1}$ ) agrava la situación. Otros aspectos, como la llegada de Inmigrantes ralentiza el fenómeno.

Pero encuestas de opinión sobre intenciones y preferencias reproductivas como la del Eurobarómetro y las realizadas por organizaciones como la OCDE o por institutos como el INE, muestran como en España la brecha entre el número ideal (deseado) de hijos por mujer y el número real de hijos, es bastante grande. Esto pone de manifiesto, por tanto, que las aspiraciones reproductivas individuales no se están viendo cumplidas por parte de las mujeres españolas, y que estas se ven truncadas por barreras socioeconómicas (Martín Patino, 2017).

A pesar de que en España el «ideal de los dos hijos»² también está arraigado, el 66,35\% de las mujeres entre 15 y 39 años así lo manifiestan, la realidad muestra que se aleja mucho de los ideales reproductivos ${ }^{3}$. Algunos estudios han vinculado esta brecha a las mujeres con estudios universitarios, ya que son las que anteponen la vida laboral a la familiar, atrasando la edad de maternidad y, con ello, acabando su periodo reproductivo con un número de hijos inferior al que deseaban.

Por ello el objetivo de este trabajo es comprobar que existe dicha brecha entre el número de hijos deseados y el número de hijos reales y estudiar las posibles razones, comportamientos individuales y/o barreras de tipo socioeconómico e institucional que pueden es-

\footnotetext{
1 Ver más: Kreyenfeld, M., \& Konietzka, D. (Eds.). (2017). Childlessness in Europe: Contexts, Causes, and Consequences. Demographic Research Monographs. doi:10.1007/978-3-319-44667-7

${ }^{2}$ El ideal de los dos hijos hace referencia a una composición familiar de dos hijos y mixta, es decir, una preferencia por al menos un niño y una niña. Ver más: Mills, M., \& Begall, K. (2010). Preferences for the sex-composition of children in Europe: A multilevel examination of its effect on progression to a third child. Population Studies, 64(1), 77-95. doi:10.1080/00324720903497081

3 Datos obtenidos de la Base de Datos de Familia de la OCDE http://www.oecd.org/social/family/database.htm
} 
tar afectando a los deseos reproductivos de las mujeres españolas. Para ello se analizará la Encuesta de fecundidad 2018 proporcionada por el INE y, en especial, el apartado de fecundidad deseada, de las mujeres de entre 45 y 55 años, cuyo periodo reproductivo ya ha finalizado. Se realizará dos modelos de regresión logística, uno para aquellas mujeres que no han tenido hijos, y otro para las que sí han tenido.

El trabajo se divide en tres partes diferenciadas: en una primera parte, se presenta el análisis del marco teórico en el que se exponen las principales teorías sobre fecundidad, se conceptualizan los principales problemas vinculados a los cambios en el ISF obtenidos de estudios previos y se contextualiza el caso español. Después se plantean las hipótesis y se explica la metodología desarrollada. Por último, aparece un breve análisis descriptivo sobre el deseo y la realidad reproductiva de las mujeres españolas entre 45 y 55 años, y se analizan los resultados de los modelos de regresión desarrollados.

\section{ANÁLISIS TEÓRICO Y CONTEXTO ESPAÑOL}

En torno a la fecundidad, giran dos grandes teorías. La primera es la Teoría de la familia desarrollada por Gary Becker (1987), la segunda, la Teoría de la segunda Transición demográfica fundamentada por Ron J. Lesthaeghe (1991) y Dirk J. Van de Kaa (1987). Por otro lado, existe una teoría que se centra en el papel de la mujer en el contexto de sociedad moderna, es la Teoría preferencial de Catherine Hakim $(1998,2000)$.

La primera es una teoría en la que Gary Becker en Tratado sobre la familia (1987) estudia los cambios de fecundidad desde un enfoque económico, analizando el coste relativo de los hijos y la interacción entre cantidad de hijos y calidad de hijos. El coste relativo de los hijos depende de los cambios del valor del tiempo de las mujeres casadas, ya que el tiempo es el componente más importante del coste total de producción y crianza de los hijos. Por ello, la capacidad de las mujeres de obtener ingresos ha sido la causa principal del aumento de participación laboral de las mujeres casadas y, por tanto, del fuerte descenso de fecundidad.

Según esta teoría, la interacción entre cantidad y calidad de hijos es la causa más importante de que el precio efectivo de los hijos aumente con la renta puesto que un aumento en la calidad, como el aumento en capital humano (inversiones en educación, salud y otros componentes), reduce la cantidad, cambiando la demanda de cantidad de hijos por la demanda de calidad de hijos. Una reducción del número de hijos por pareja puede aumentar el peso de sus hijos en la generación siguiente si esto permite que la pareja invierta más en la educación, formación y potencial de cada hijo superviviente. Cada familia maximiza una función de utilidad cuyos argumentos son la cantidad de hijos, el gasto en cada hijo (calidad) y las calidades de otras mercancías. El desarrollo económico afecta a la fecundidad y la calidad de hijos, no solo porque aumentan las rentas de las familias, 
sino también porque aumentan las tasas de rendimiento de las inversiones en educación y otras formas de capital humano. En definitiva, la reducción de la fecundidad es el resultado de un aumento del precio de la cantidad de hijos y de la interacción entre cantidad y calidad de hijos.

Por tanto, existe una relación inversa en las mujeres entre el nivel educativo y la ocupación, y el hecho de tener un primer hijo. Es decir, una mayor inversión en educación y al mundo laboral hace que aumente el coste de oportunidad de la maternidad en términos de salarios no ingresados y de acumulación perdida de capital humano. Posponer la maternidad es una estrategia para disminuir el coste de oportunidad que les supondría abandonar su carrera profesional. A mayor edad se espera que haya alcanzado una posición y estabilidad laboral lo que supone que los riesgos asociados a la interrupción de su carrera después de la primera maternidad son menores. (Vidal-Coso \& Miret-Gamundi, 2017).

En cambio, la Segunda Transición demográfica explica la evolución de la fecundidad desde una perspectiva sociológica, como resultado de los cambios de mentalidad y de valores que, a partir de la segunda mitad del siglo xx, supusieron la pluralización de las formas de vida familiares. La principal característica de la Segunda Transición Demográfica es la disminución de la fecundidad por debajo de los niveles de reemplazo generacional, 2,1 hijos por mujer, que asegura que los nacimientos y las muertes estarán en balance y que la población permanece estacionaria a lo largo del tiempo, a un nivel muy por debajo del reemplazo (Van de Kaa, 1987).

Según Lesthaeghe (1991) esta transición se divide en tres fases. La primera fase se inició en 1955 y se caracterizó por la aceleración del aumento del número de divorcios, disminuyendo la duración de los matrimonios; por la finalización del baby-boom; y porque se produjo una caída de la fecundidad en todas las edades, por debajo del nivel de reemplazo generacional, lo que coincidió con la revolución contraceptiva. La segunda fase se dio entre 1970 y 1985, y su principal característica fue el aumento de la cohabitación antes del matrimonio lo que supuso que en algunos países compensase el descenso de la nupcialidad e implicase un aumento considerable de nacimientos fuera del matrimonio. La última fase se dio a partir de mediados de los ochenta en adelante y destacó por una recuperación de la tasa de fertilidad en mujeres mayores de 30 años.

El argumento llevado en torno a la segunda transición demográfica se hace desde una interpretación teórica posmoderna y posmaterialista en el que la disminución del número de hijos, la caída de las tasas de nupcialidad, la inestabilidad creciente de las relaciones, la aparición de nuevas formas familiares alejadas de la familia nuclear y la expansión de la cohabitación, representan la difusión de valores que promueven orientaciones y estilos de vida individualistas, la búsqueda de identidad y la realización personal, por encima de los compromisos y los vínculos a largo plazo, la religiosidad o la sumisión a los prejuicios (Esping-Andersen et al., 2013). 
Por último, se encuentra una teoría individualista desarrollada por Catherine Hakim, denominada Teoría preferencial (Hakim, 2000), que estudia la actitud de la mujer a la hora de elegir entre participar en el mercado laboral o ser madre. Este enfoque se sustenta en cuatro principios (Hakim, 1998). El primero, el nuevo escenario del mercado laboral, que ha aportado a la mujer diferentes oportunidades y opciones, y que está marcado por cinco acontecimientos que han surgido a lo largo del siglo xx: la revolución anticonceptiva que proporcionó a las mujeres un control confiable e independiente de su sexualidad; segundo la revolución de igualdad de oportunidades, facilitando el acceso a prácticamente todas las esferas del mercado laboral. El tercero, la expansión de las ocupaciones de cuello blanco, que son mucho más atractivas para las mujeres que la mayoría de las ocupaciones de cuello azul. En cuarto lugar, la creación de puestos de trabajo secundarios, que no se centran en darle prioridad al trabajo remunerado a expensas de otros intereses de la vida. Y, por último, la importancia de las actitudes, los valores y las preferencias personales en las opciones de estilo de vida de las sociedades modernas. El segundo principio hace referencia a la heterogeneidad de las mujeres en sus preferencias y prioridades sobre la elección entre la vida familiar y el empleo. Hakim se centra en tres grupos: el primero hace referencia a la mujer "centrada en el trabajo», este grupo lo forman mujeres sin hijos cuya prioridad fundamental es el trabajo profesional. El segundo grupo es el de la mujer "adaptativa», incluye a aquellas mujeres que quieren compatibilizar trabajo y familia, sin dar prioridad al trabajo profesional. El tercer grupo es el de la mujer "centrada en el hogar», mujeres que prefieren no trabajar y para las que la vida familiar y los niños son sus principales prioridades. El tercer principio viene del segundo, pues la heterogeneidad de las preferencias y prioridades de las mujeres crea intereses en conflicto entre los distintos grupos de mujeres. Estos intereses en conflicto de las mujeres han dado una gran ventaja a los hombres, cuyos intereses son genéticos, esta es una causa del patriarcado y su éxito desproporcionado. Por último, el cuarto principio, dice que la heterogeneidad de las mujeres es la causa principal de las respuestas variables de las mujeres a las políticas sociales en el nuevo escenario de la sociedad moderna.

Actualmente España se encuentra dentro del nivel de Lowest-low-fertility, nivel que caracteriza a aquellas sociedades con un ISF por debajo de 1,3, lo cual provoca una disminución de 1,5\% anual del tamaño de la población. Según (Kohler et al., 2004) este proceso demográfico se debe a la combinación de cinco factores, que son: los cambios demográficos en el periodo de fertilidad, debido al aplazamiento de la maternidad que ha reducido el nivel de fecundidad asociado a cada cohorte. La situación económica y social, provocando una respuesta racional en los individuos sobre la posposición de la maternidad. Los procesos de interacción social que afectan al periodo reproductivo provocando una respuesta en el conjunto de la población haciendo que los cambios socieconómicos puedan explicar el rápido proceso de aplazamiento de la edad de maternidad asociado al fenómeno demográfico lowest-low fertility. El marco institucional, que en los países del sur, centro y este de Europa han favorecido un bajo nivel de fecundidad. Y, por último, la interacción post- 
momen-quantum ${ }^{4}$, que ha agravado las consecuencias en dichos entornos institucionales y han causado una gran reducción de la fecundidad en los países caracterizados por el lowest-low fertility en los que se retrasa la maternidad.

Asimismo, a la hora de hablar de fecundidad hay que tener en cuenta el control de natalidad, que hace referencia al control voluntario de las mujeres sobre el número de hijos, su espaciamiento y la fecha en la que se desea el primer y el último embarazo. Esta es una decisión racional en las que las mujeres buscan una mejor salud de ellas mismas y de los hijos, una disminución de hijos no deseados o ilegítimos, una mejor situación económica que permita un mejor nivel de vida e inversión en educación de los hijos, un mayor control sobre su cuerpo, lo cual es un buen reflejo de la sociedad (Miguel, 1980).

Otro de los fenómenos demográficos que tiene especial relevancia en España es la infecundidad, es decir, aquellas mujeres que sobrepasan los 45 años, edad con la que finaliza el periodo reproductivo, y no han sido madres. En los últimos años, ha aumentado considerablemente, pasando de poco más del 12\% en 1999 hasta llegar al 19\% en $2017^{5}$ siendo especialmente alta entre aquellas mujeres con un alto nivel de estudios y en aquellas que nunca se han casado (Reher \& Requena, 2018). Dentro de la infecundidad, Devolver y Domingo (2016) distinguen cinco grupos de mujeres que difieren en las causas o motivos. En primer lugar la infecundidad primaria, que es aquella que se da por razones biológicas. Segundo, la infecundidad deseada, mujeres que no quieren ser madres. Tercero, la infecundidad normativa, mujeres que quieren y pueden pero se consideran muy jóvenes para ello. Cuarto, la infecundidad transitoria, mujeres que optan por posponer su maternidad porque las condiciones materiales y/o familiares no son las adecuadas. Por último, la infertilidad sobrevenida, que hace referencia a las mujeres, especialmente a partir de los 35, que cuando deciden ser madres no pueden debido a que ya no son fértiles porque tienen demasiada edad.

A pesar de la dificultad a la hora de medir cada una de ellas, todo apunta a que aquellas causas que tienen que ver con el retraso de la edad de la maternidad y las que rodean a las circunstancias económicas y sociales son las que más afectan a este fenómeno, lo que indica la alta dificultad de los más jóvenes de satisfacer sus deseos reproductivos y realizar sus proyectos (Bernardi \& Requena, 2004). Además, la infecundidad involuntaria ha descendido en los últimos años debido a las técnicas de reproducción asistida, siendo España uno de los países europeos con mayor utilización de estas técnicas, estimándose que aproximadamente un 3\% del total de todos los nacimientos han sido concebidos mediante reproducción asistida (Castro \& Seiz, 2014).

\footnotetext{
${ }^{4}$ Ver más en página 667 Kohler, H. P., Billari, F. C., \& Ortega, J. A. (2002). The emergence of lowest-low fertility in Europe during the 1990s. Population and development review, 28(4), 641-680.

${ }^{5}$ Fuente: INEbase (https://www.ine.es/).
} 
La relación entre infecundidad y descendencia final no es lineal, pero durante los últimos años esta relación se ha estrechado debido al aumento de la infecundidad y, no tanto, a la reducción del número medio de hijos de mujeres que han sido madres (Esteve et al., 2016). Contrario a esto, Esping-Andersen, menciona que la infecundidad se da en un porcentaje muy reducido, ya que la mayoría de las mujeres tienen hijos, pero muchas de ellas solo tienen uno (Esping-Andersen et al., 2013)

Numerosas investigaciones se han realizado en torno a la fecundidad en España en los últimos años, sus principales aportaciones han girado en torno a numerosos factores demográficos como estructurales que se presentan a continuación.

La edad media del primer hijo, entendido como un desplazamiento y no como un retraso, es el factor demográfico que opera más directamente con el ISF, ya que el envejecimiento del calendario de la maternidad conlleva una preponderancia creciente del primer rango en detrimento de todos los demás, es decir, cuanto más se tarde en tener el primer hijo más probabilidades hay de no tener un segundo, y muchas más de no tener un tercero, y así sucesivamente(Delgado, Zamora López, \& Barrios, n.d.). Además, a medida que aumenta la edad aumenta la perdida de fertilidad (Esping-Andersen et al., 2013). En España, la edad media de la primera maternidad en la década de los 90 era de 26,8 años, a principios del siglo xxi era de 29,2 años y actualmente es de 30,96.

Otro factor demográfico que incide en la fecundidad es el matrimonio, cada vez se casan menos y más tarde. Desde el año 2000 hasta el año 2017, la edad del primer matrimonio ha aumentado cinco años, situándose en los 32,2 años, siendo una de las más altas de Europa, asimismo la tasa de nupcialidad ha caído desde los 5,4 matrimonios por mil personas en el año 2000 a los 3,7 en 20177. Como consecuencia de estas tendencia, la proporción de mujeres en las edades reproductivas modales (el grupo de edad de 30 a 34 años) que se encuentran casadas ha descendido notablemente en España: de 81,4\% en 1980 a 51,1\% en 2011, lo que tiene consecuencias negativas en la formación familiar y, por tanto, en la descendencia final (Castro \& Seiz, 2014).

También cabe resaltar la influencia de la población inmigrante ya que se caracterizan por pertenecer a edades reproductivas con un calendario de maternidad más temprano que el de las mujeres españolas y con un promedio de hijos mayor al de los españoles, lo que se traduce en tasas de fecundidad más altas(Castro Martín \& Rosero-Bixby, 2011). En España, en el año 1996, solo el 3,3\% de los nacimientos eran de madres extranjeras, 20 años después, suponían el 20,7\%. En el año 2012, casi uno de cada cuatro nacimientos (el 23\%) en España tenía un padre o una madre de nacionalidad extranjera (Castro \& Seiz, 2014).

\footnotetext{
${ }^{6}$ Fuente: INEbase (https://www.ine.es/).

${ }^{7}$ Fuente Eurostat (ec.europa.eu/eurostat/data/database).
} 
La estabilidad laboral supone una obligación previa a la formación de la familia. El escenario español no es óptimo para tener hijos: los jóvenes no disponen de trabajo, ni de una vivienda propia. Martín Patino (2017) afirma que la decisión de tener hijos o no se aplaza en tiempos de crisis económica e incertidumbre laboral y, en el caso español el desempleo y la precariedad laboral han tenido un fuerte efecto sobre la fecundidad.

Un contexto institucional en el que existen políticas públicas orientadas a la compatibilización de la vida familiar y laboral, tiene consecuencias en la edad y el número de nacimientos, debido al coste de oportunidad. En España, dicho contexto institucional es limitado, lo que tiene un efecto negativo en la fecundidad (Mínguez, 2012).

Además, estudios como el de Esping Andresen et al. (2013) muestran que en España, la diferencia entre el número de hijos deseados y el número de hijos reales es notablemente acusada en las mujeres, a pesar de que el ideal de los dos hijos sigue presente. Uno de los factores que marcan esta diferencia es que las mujeres con estudios universitarios posponen su maternidad, algo característico de los países del sur de Europa donde las mujeres encuentran dificultades a la hora de compaginar la vida laboral con la vida familiar debido a unos contextos laborales, institucionales y familiares no apropiados, en los que el hecho de criar y mantener a los hijos supone, en especial para las mujeres con estudios superiores, un elevado coste de oportunidad de trabajo (Mínguez, 2012).

Por ello, autoras como Sampedro, Gómez, y Montero ven el hecho de posponer la maternidad, no como el resultado de un fracaso o fallo trascendental, sino que como «el resultado lógico de unas trayectorias vitales que se estructuran de una forma muy definida: finalización de los estudios, logro de una situación laboral estable y de un cierto capital de experiencia profesional, disfrute de un tiempo de consumo y realización personal (viajes...), consolidación de una situación económica que permita hacer frente a inversiones importantes como la compra de una vivienda, y, por fin, formación de una familia, con la llegada de los hijos» (pp31, 2002).

España puede considerarse un caso particular para el análisis de la fecundidad y el comportamiento reproductivo de las mujeres. En primer lugar y como se ha visto, debido al ISF se sitúa en uno de los más bajos del mundo, 1,3 hijos por mujer. Por tanto, si la tasa de fecundidad se sigue manteniendo en niveles tan bajos acarreará importantes consecuencias con grandes problemas a largo plazo. Por un lado, la población total se reducirá debido a la incapacidad de reemplazarse, se estima que en 2100 la población española será de entre diez y quince millones de personas, un 25\% menos de la población actual (Esping-Andersen et al., 2013). Por otro lado, la población anciana aumentará, por lo que el número de personas jóvenes que tenga que hacerse cargo de las personas mayores será escasa. Esto acarreará problemas de salud relacionado con los cuidados, económicos por la incapacidad de mantener las pensiones y las políticas y marcos institucionales tendrán que orientarse a una nueva sociedad envejecida, que conllevará combatir nuevos desafíos. 
En segundo lugar, la infecundidad es un fenómeno demográfico que cada vez tiene más relevancia en el contexto español debido a su progresivo aumento. Se estima que el 25\% de mujeres nacidas en los 70 llegará al final de su vida fértil (45 años) sin hijos, mientras que las nacidas en los años 55, suponen el 10\% ${ }^{8}$. Dado que las prácticas de reproducción asistida avanzan progresivamente, la infecundidad causada por problemas biológicos cada vez supone un menor porcentaje. Por esta razón, es importante analizar cuáles son los factores que determinan o los motivos que llevan a las mujeres a acabar su periodo reproductivo sin hijos.

Por último, debido a la brecha que hay entre el número ideal y el número real de hijos entre las mujeres. El ideal de los dos hijos sigue estando presente en España pero las expectativas de las mujeres no se ven cumplidas, según el Eurobarómetro 2011, el número medio ideal de hijos entre mujeres de 20 y 49 años era de 2,13, mientras que el real era de 1,22. Por lo tanto, esta variación, en el caso de las mujeres españolas es de casi un hijo, un dato importante y una diferencia de las más altas de Europa. Esto lleva a preguntarse cuáles son los motivos o razones que llevan a que las mujeres no vean cumplidas sus expectativas reproductivas.

Como se ha visto en investigaciones previas, este problema se da con mayor preponderancia en las mujeres con estudios universitarios. Si el patrón se sigue repitiendo, es decir, si las mujeres con un nivel de estudios superior siguen coincidiendo, en mayor medida, con las mujeres que no desean tener hijos o que ven incumplidas sus expectativas reproductivas al tener un número de hijos menor del deseado, la situación en España se agravará. Esto se debe a que desde el año 2000 hasta el año 2018 el porcentaje de mujeres con educación universitaria ha pasado de ser del $21,1 \%$ al 36,4\% ${ }^{9}$, lo que supone un aumento del 15\% de mujeres con un nivel de estudios universitarios y, en concordancia, la reducción de mujeres con una educación baja y media que son, comúnmente, las que tienen un número mayor de hijos.

\section{METOdología}

El presente análisis, partiendo de los fundamentos teóricos planteados anteriormente, tiene como objetivo conocer aquellos factores que hacen que las mujeres no vean cumplidas sus expectativas reproductivas en España, partiendo de las siguientes hipótesis.

H1: una posposición de la primera maternidad tiene una relación negativa con el número de descendencia final, es decir, a medida que aumenta la edad del primer hijo, disminuye el número de hijos, lo que ocasiona la diferencia entre el número de hijos deseados y el real.

\footnotetext{
${ }^{8}$ Fuente: CED a partir de datos del INE.

${ }^{9}$ Fuente: INEbase (https://www.ine.es/).
} 
H2: aquellas mujeres con un nivel educativo superior son las que toman la decisión de no ser madres, en contraste a las mujeres con un nivel educativo inferior, lo que provoca, en mayor medida, que no vean cumplidas sus expectativas reproductivas.

Para ello se ha escogido la Encuesta de fecundidad de 2018 del Instituto Nacional de Estadística, en este caso, la realizada a mujeres de 18 a 55 años de todo el territorio español. Para este estudio, se ha seleccionado a las mujeres entre 45 y 55 años, ambos incluidos, es decir, a aquellas mujeres cuya vida reproductiva ha finalizado en el momento en el que se recogieron los datos, lo que supone una muestra de 5367 casos.

Esta encuesta, además de contar con información sobre características socio-demográficas, también incluye un apartado de fecundidad deseada. Esto permite conocer el número exacto de hijos deseados y de hijos reales y, por tanto, mirar si hay una brecha entre los mismos, tanto en mujeres que sí han tenido hijos, como para las que no.

Con el fin de confirmar las hipótesis anteriormente planteados, se llevarán a cabo dos modelos de regresión logística binomial (López-Roldá \& Fachelli, 2016), técnica estadística multivariable que permite pronosticar los valores de una variable dependiente categórica dicotómica a partir de una o más variables explicativas independientes, también llamadas covariables. Mediante esta técnica es posible identificar cuáles son las características o factores que producen la diferenciación entre ambos grupos ya que el objetivo del análisis multivariable es la determinación del mejor modelo explicativo, lo que significa comprobar la estimación de las diferentes variables independientes propuestas inicialmente en el modelo de regresión logística y escoger aquellas que mejor explican la variable dependiente.

Los modelos implementados en este documento son dos. El primero hará referencia a las mujeres que no tienen hijos. Las variables independientes en este primer modelo son: edad que tenía cuando contrajo matrimonio, estado civil (EC, dividido en: Soltera, Casada, Viuda, Separada y Divorciada), nacionalidad (NAC cuyas categorías son: Española, Extranjera, Española y otra), grado de urbanización del municipio de residencia (DEGURBA, categorizada en: Urbano, Intermedio y Rural) religión (Religion: dividido en creyentes y No creyentes), nivel de estudios (primario, secundario, postsecundarios y universitarios) y motivos por los que no han tenido hijos, para ver si hay correlación con la variable dependiente: «Si deseaba hijos» que se divide en dos grupos: las que sí deseaban tener hijos y las que no.

El otro modelo, se centra en las mujeres con hijos. Las variables independientes seleccionadas son: estado civil (EC, dividido en: Soltera, Casada, Viuda, Separada y Divorciada), nacionalidad (NAC cuyas categorías son: Española, Extranjera, Española y otra), grado de urbanización del municipio de residencia (DEGURBA, categorizada en: Urbano, Intermedio y Rural), religión (Religion: dividido en creyentes y no creyentes), nivel de estudios (primario, secundario, postsecundarios y universitarios), edad del primer hijo y edad que tenía 
cuando se casó. Con estas variables, se pretende conocer si hay una influencia en la variable dependiente «número de hijos real es menor del deseado» cuyas respuestas se dividen entre las mujeres que sí han tenido menos hijos de las que les hubiese gustado tener y las que no.

Hay que tener en cuenta que estos modelos cuentan con cierta limitación metodológica. Esto se debe a que en el mismo modelo se han incorporada tanto variables micro como variables macro para estudiar la fecundidad. Las variables a nivel micro, que hacen referencia al comportamiento individual, son el nivel de estudios, la religión, el estado civil o la nacionalidad. Y las variables macro, el grado de urbanización del municipio de residencia o, indirectamente, la variable motivos que introduce valores que hacen referencia a factores estructurales como la situación laboral y económica.

\section{RESULTADOS}

Como ya se ha visto, el número de hijos por mujer en España desde hace dos décadas está muy por debajo del reemplazo generacional. Pero esta fecundidad real se aleja mucho de la fecundidad deseada. Para ello, se va a detallar el porcentaje de hijos reales y el deseado de mujeres entre 45 y 55 años ofrecido por la Encuesta de Fecundidad de 2018.

Gráfico 1. Porcentaje de número deseado y número real de hijos de mujeres entre 45 y 55 años

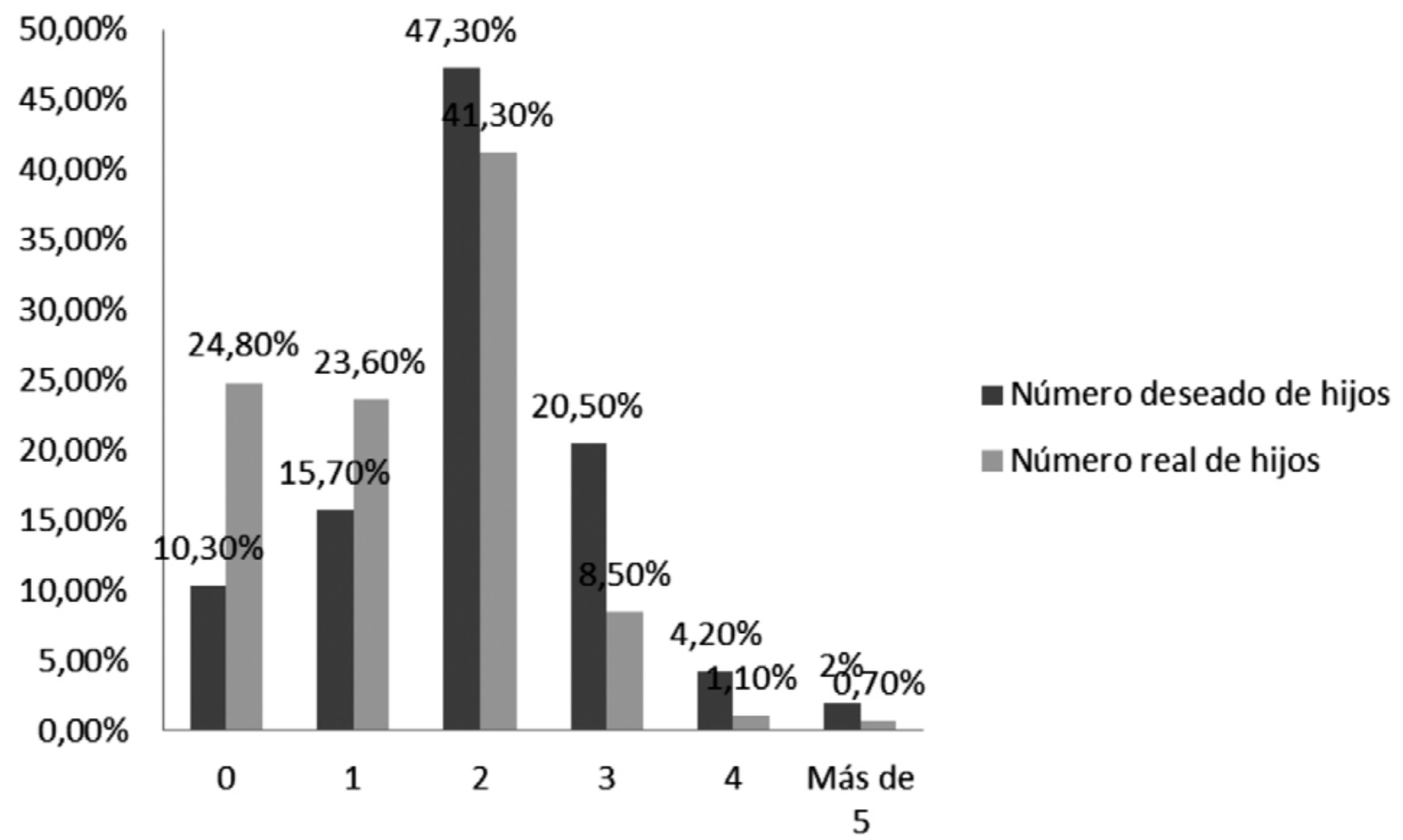


De las 5367 mujeres de entre 45 y 55 años, el número medio real de hijos es de 1,4, mientras que el número medio deseado de hijos es de dos hijos. A su vez, dos hijos es la opción más común tanto real, como deseado, pues el 41,3\% de las mujeres tienen dos hijos y el $47,3 \%$ de mujeres deseaba tener dos hijos.

Las grandes diferencias se dan cuando se refiere al hecho de no tener hijos, ya que el porcentaje de mujeres que no tiene ningún hijo es del 24,8\%, es decir, una de cada cuatro mujeres, mientras que tan solo el 10,3\% deseaba no tener hijos.

Otra gran diferencia se da en que el 20,5\% de las mujeres deseaba tener tres hijos, pero únicamente el 8,5\% de las mujeres tiene tres hijos. El número real es mayor que el deseado cuando se trata de tener un único hijo, con el 23,6\% y el 15,7\% respectivamente.

Esta brecha entre el número deseado de hijos y el número real de hijos se debe, principalmente, a que de las 1.030 mujeres que no han tenido hijos, el 47,4\% sí deseaba haberlos tenido y de las 4.334 mujeres que sí han tenido hijos, el 29\% afirma que ha tenido un número menor de hijos de los que deseaba. En definitiva, casi la mitad de las mujeres sin hijos, sí les hubiera gustado tener hijos y una tercera parte de las mujeres que han tenido hijos, han tenido un número inferior del que deseaban.

\section{Mujeres sin hijos}

El primer modelo de regresión logística llevado a cabo es empleado en mujeres que no han tenido hijos (Tabla 1), el objetivo es pronosticar el valor «Sí deseaban tener hijos» de la variable dependiente, a partir de una serie de variables independientes, ya que casi el $48 \%$ de las mujeres si deseaban haber tenido hijos.

Este modelo, mediante El Pseudo R cuadrado de Nagelkerke, indica que el modelo tiene la capacidad de predicción de 0,545 de la variable dependiente, lo que significa que es un modelo bastante aceptable. 
Tabla 1. Regresión mujeres sin hijos

Variable dependiente: deseo de tener hijos en mujeres de 45 a 55 años.

(Categoría de referencia: sí deseaban)

Sig.

Estado civil

Soltera (cat.reg)

Casada

Separada

Divorciada

Edad a la que contrajo matrimonio

\section{Nacionalidad}

Española (cat.ref)

Extranjera

Española y otra

Grado urbanización del municipio

Urbano (cat.ref)

Intermedio

Rural

\section{Religión}

Creyente (cat.ref)

No creyente

0,326

Nivel de estudios

1,169

Estudios primarios

Estudios secundarios

0,414

0,413

Estudios postsecundarios

Estudios universitarios (cat.ref)

Motivos por los que no han tenido hijos

0,778

$-0,001$

$-0,379$

$-0,1$

Dificultad conciliación vida familiar y laboral (cat.ref)

No quería ser madre

No ha tenido pareja

Falta de recursos económicos

Por problemas de salud

Era demasiado joven

Demasiada edad para tener hijos

\section{Constante}

$-3,468$

1,341

$-0,504$

1,255

$-21,78$

$-0,406$

$-2,02$

$\mathrm{N}$ total

270

Pseudo R2 de Nagelkerke
2,177

0,999

0,328

0,999

0,77

0,288

830902946,6

568897953,6

1,034

0,107

0,619

0,621

0,684

0,333

0,905

0,828

$1,386 \quad 0,466$

0,108

3,219

0,016

1,513

0,371

1,511

0,432

0

0,031

0

3,823

0,217

0,604

0,464

3,508

0,045

0,999

0,667

0,559 
En el modelo de regresión, la Beta, indica la dirección de la influencia de las variables independientes sobre la variable dependiente, en este caso, sobre las mujeres que no han tenido hijos, pero que sí deseaban tenerlos. La variable independiente «Edad a la que contrajo matrimonio» indica que a medida que aumenta esta, disminuye la probabilidad de que se de la variable dependiente, es decir, de que las mujeres sin hijos sí deseen tener hijos. El «Estado civil» es otra de las variables independientes, la variable categórica de referencia es Soltera, lo que indica que respecto a ella el hecho de estar casada, divorciada o separada hace que aumente la probabilidad de que la mujer sí desee tener hijos. La «Nacionalidad», incluida en el análisis, toma la categoría de referencia ser española, lo que hace que tener nacionalidad extranjera aumente las probabilidades de que se de la variable dependiente, pero de que disminuya si la nacionalidad es española y otra. En cuanto al "Grado de urbanización del municipio de residencia», el vivir en un sitio rural o intermedio reduce la probabilidad de que las mujeres deseen tener hijos, respecto a las mujeres que viven en municipios urbanos. La variable «Nivel de estudios» indica que, aquellas mujeres con estudios primarios, estudios secundarios y estudios postsecundarios, aumenta la probabilidad de que hayan deseado tener hijos, respecto a las mujeres con estudios superiores. Respecto a la «Religión», el hecho de ser no creyente aumenta la probabilidad de que las mujeres sin hijos deseen hijos, en comparación a las mujeres creyentes.

Los «Motivos» que las propias mujeres contestan cuando se les pregunta por qué no han tenido hijos es otra de las variables independientes. La categoría de referencia de los motivos es la dificultad a la hora de compatibilizar la vida familiar y laboral. Respecto a esta variable, el motivo de no querer ser madre disminuye la probabilidad de la variable dependiente, al igual que la falta de recursos económicos, el ser demasiado joven y tener demasiada edad para ser madre. En cambio, aumenta la probabilidad de que se de la variable dependiente, respecto a la misma categoría de referencia, cuando se dan los motivos de que no ha tenido una pareja o por problemas de salud.

Pero la significación de las variables independientes, indica que solo es significativa, es decir, que hay relación con la variable dependiente, la variable motivos, puesto que es la única con un valor menor de 0,05. En concreto, los motivos respecto a no querer ser madre y a problemas de salud. Además, aunque la variable nivel de estudios no es en su conjunto significativa, si lo es el valor de estudios primarios. 
INGURUAK [67] | 2019 | 35-58

Factores que interfieren en el incumplimiento de las expectativas reproductivas de las... | Aidée Baranda Ortiz

Mujeres con hijos

Tabla 2. Regresión mujeres con hijos

Variable dependiente: mujeres entre 45 y 55 años que han tenido menos hijos de los que deseban

$$
\beta
$$

$\operatorname{Exp}(\beta)$

Sig.

\section{Estado civil}

0,569

Soltera (cat.reg)

Casada 1,04

2,829

0,362

Separada

19,269

233487196,4

0,999

Divorciada

$-0,299$

0,741

.851

Edad a la que contrajo matrimonio

0,012

1,012

0,609

Edad a la que tuvo el primer hijo

0,115

1,121

0,01

Nacionalidad

0,32

Española (cat.ref)

Extranjera

$-0,91$

0,403

0,131

Española y otra

$-0,17$

0,843

0,83

Grado urbanización del municipio

0,267

Urbano (cat.ref)

Intermedio

$-0,476$

0,621

0,161

Rural

0,107

1,113

0,83

\section{Religión}

Creyente (cat.ref)

No creyente

0,195

1,261

0,673

Nivel de estudios

0,941

Estudios primarios

$-0,091$

0,855

Estudios secundarios

$-0,132$

0,913

0,782

Estudios postsecundarios

0,14

0,876

0,783

Estudios universitarios (cat.ref)

Constante

$-1,476$

$\mathrm{N}$ total

826

Pseudo R2 de Nagelkerke

0,111

Este modelo (Tabla 2), con el objetivo de predecir cuales son las variables independientes que inciden en el hecho de que las mujeres que han tenido hijos, tengan un número de hijos menor al número de los que deseaban, es significativo. Mediante el proceso de regresión logística, el Pseudo R2 es de 0,11, lo que indica que el modelo es aceptable y con capacidad de predicción. 
Respecto a la incidencia de las variables independientes en la variable dependiente, está Beta, que indica la dirección de la relación entre la variable independiente y la variable dependiente. En este modelo se ve que a medida que aumenta la variable «Edad a la que se tuvo el primer hijo», aumenta la probabilidad de se tenga menor número de hijos del que se desea. Lo mismo pasa con la «Edad a la que se casó», al aumentar, aumenta la probabilidad de que el número de hijos reales sea menor del que deseaba. En cuanto al «Estado civil», la categoría de referencia es el primer valor: soltera. La probabilidad de la variable dependiente aumenta cuando es casada y separada, y disminuye cuando es divorciada. La variable independiente "Nacionalidad», tiene como categoría de referencia a la nacionalidad española, respecto a ella, el hecho de tener una nacionalidad extranjera o española y otra, hace que disminuya la probabilidad de la variable dependiente. El «Grado de urbanización del municipio de residencia» es otra de las variables independientes, en este caso, la categoría de referencia es urbano, por lo que se puede decir que un grado de urbanismo intermedio hace que disminuya la probabilidad de que el número de hijos reales sean menores al número deseado, pero que aumente la probabilidad si el municipio es rural. En torno a la variable independiente "Nivel de estudios», cuya categoría de referencia es Estudios universitarios, indica que las mujeres con estudios primarios y secundarios tienen menor probabilidad de tener un número de hijos por debajo del deseado, mientras que si son estudios postsecundarios la probabilidad aumenta. Respecto a la religión, el hecho de ser creyente, categoría de referencia, hace que aumente la probabilidad de que el número de hijos real sea menor al número de hijos deseado cuando las mujeres son no creyentes.

Pero observando la Significación de cada una de las variables, se observa que únicamente la variable independiente «Edad del primer hijo» explica la variable dependiente, al tener un valor menor de 0,05.

Tabla 3. Motivos por los que han tenido menos hijos de los que deseaban

\begin{tabular}{|c|c|c|c|c|c|c|c|}
\hline $\begin{array}{l}\text { Problemas de } \\
\text { salud }\end{array}$ & $\begin{array}{l}\text { Falta de recursos } \\
\text { económicos }\end{array}$ & $\begin{array}{l}\text { Dificultad } \\
\text { conciliación vida } \\
\text { laboral y familiar }\end{array}$ & Demasiada edad & $\begin{array}{c}\text { No tenía una } \\
\text { pareja } \\
\text { adecuada }\end{array}$ & $\begin{array}{l}\text { Su pareja ya tenía } \\
\text { o no queria más }\end{array}$ & $\begin{array}{c}\text { No queria tener } \\
\text { más hijos }\end{array}$ & $\begin{array}{l}\text { Dificultad para } \\
\text { adoptar }\end{array}$ \\
\hline $27 \%$ & $23 \%$ & $20,90 \%$ & $13,40 \%$ & $7,60 \%$ & $5,80 \%$ & $1,50 \%$ & $0,90 \%$ \\
\hline
\end{tabular}

Fuente: elaboración propia a partir de la Encuesta de Fecundidad 2018 del INE.

Para conocer más sobre por qué el número deseado de hijos no coincide con el real, se ha realizado una tabla de frecuencias. En ella se puede observar que de las 1216 mujeres que contestaron a la pregunta, el 27\% respondió que era por problemas de salud. Seguido de este motivo, se encuentra la falta de recursos económicos, con un $23 \%$ y la dificultad a la hora de conciliar la vida laboral y familiar con el 20,9\%.

Algo menos de importancia tiene tener demasiada edad con el 13,4\%; no tener una pareja adecuada, 7,6\%; y que su pareja ya tenía hijos o no quería más, 5,8\%. Los motivos más irre- 
levantes son la dificultad para adoptar con el 0,9\% y que no querían tener más hijos con el $1,5 \%$ de los casos.

\section{CONCLUSIONES}

El análisis presentado en este trabajo ha permitido, en primer lugar, demostrar que existe una gran brecha entre el número de hijos deseados y el número de hijos reales. En este caso, como ya se ha comentado, el grupo de mujeres escogido para el estudio fueron las mujeres entre 45 y 55 años, que ya habían finalizado su vida reproductiva y, por tanto, no se podían dar cambios. Dichas mujeres han manifestado, de media, que les hubiera gustado tener dos hijos, mientras que la realidad se aleja mucho, ya que han tenido 1,4 hijos por mujer. Por tanto, el ideal de los dos hijos sigue arraigado entre las mujeres españolas, pero sus expectativas no se ven cumplidas.

Los modelos de regresión logística empleados, aunque cuenten con la limitación de mezclar variables tanto micro como macro, son significativos. En cuanto a las mujeres que no han tenido hijos, cabe destacar que casi el $48 \%$ sí deseaba haber tenido, por lo que a la hora de buscar cuales eran las razones que hacían que casi la mitad de las mujeres no vieran cumplidas sus expectativas reproductivas, se ha visto que las mujeres con estudios primarios sí que deseaban haber tenido hijos respecto a las mujeres con estudios universitarios. Además, dentro de los motivos que las propias mujeres daban al preguntarlas el por qué no habían tenido hijos, el hecho de carecer recursos económicos tiene una alta correlación con no haber satisfecho sus deseos. Por otro lado, cabe destacar que no haber querido tener hijos es un motivo bastante relevante a la hora de no tenerlos como cabía esperar, por lo que hay un alto porcentaje de infecundidad voluntaria dentro de las mujeres sin hijos.

Dentro del grupo de mujeres que sí han tenido hijos, el 30\% han tenido menos hijos de los que deseaban. El modelo de regresión logística binaria utilizado para ver qué factores hace que se produzca este hecho muestra que el hecho de atrasar la primera maternidad sí tiene consecuencias, las mujeres acaban teniendo menos hijos de los que deseaban.

Además, se ha visto que al igual que en el hecho de no tener hijos, la falta de recursos tiene una gran importancia, pues el $23 \%$ de las mujeres dice que no ha tenido más hijos por este motivo. A esto hay que añadirle que casi el 21\% no tuvo más hijos por la imposibilidad de compatibilizar la vida familiar con la laboral.

Los motivos referidos a problemas de salud, con un porcentaje del $27 \%$, entra en conflicto con el marco teórico y los estudios realizados en torno a la fecundidad, los cuales mencionan que la infecundidad se ha reducido por los avances médicos y cada vez los problemas de salud tienen menos relevancia. 
En cambio, tanto la teoría económica de la fecundidad de Gary Becker como la teoría preferencial de Hakim se ven confirmadas al observar que el hecho de la incorporación de la mujer al mercado laboral hace que las mujeres pospongan la maternidad, siendo el fenómeno demográfico que más relación guarda con el hecho de que caiga la tasa de fecundidad, pues el coste de oportunidad es alto a la hora de tener un hijo ya que interfiere negativamente en la carrera profesional de las mujeres. La falta de recursos económicos también es importante en esta teoría, el hecho de no contar con capital económico hace que no se pueda invertir en la calidad de la crianza de un hijo y, en consecuencia, el número de hijos se reduce o desaparece. En la teoría que defiende Hakim, de las decisiones que tiene que hacer la mujer en la actualidad respecto a la vida familiar y a la vida laboral, la categoría de «mujeres centradas en el hogar» se puede presenciar en este trabajo, ya que la incompatibilidad de la vida familiar y laboral hace que la tasa de fecundidad en España sea baja.

El estado civil no es significativo a la hora de no tener hijos o no tener hijos, así que como la segunda transición expone, ha habido un cambio de creencias y crisis de valores que indican que el hecho de estar casada deja de ser un prerrequisito para la formación de la familia. Prueba de esto también es la no diferenciación entre las mujeres creyentes y no creyentes, ya que no se ve disparidades en el comportamiento de ambos grupos de mujeres.

El nivel de estudios, variable que ha sido bastante importante en las investigaciones previas llevadas a cabo en torno a la fecundidad en el contexto español, en este trabajo solo tiene relevancia directamente con la variable dependiente en las mujeres sin hijos. Pues son las mujeres con estudios primarios, respecto a las mujeres con estudios universitarios, las que sí deseaban haber tenido hijos. Pero como pudimos ver en la primera parte del trabajo, el nivel educativo puede estar relacionado indirectamente con otras variables, como la posposición del matrimonio, del retraso de la edad del primer hijo o que son las mujeres que más peso le dan a la vida profesional en comparación a las mujeres con niveles de estudios más bajos.

El análisis, por tanto, muestra que en el actual panorama español las mujeres no están viendo cumplidas sus expectativas reproductivas. Cada vez un porcentaje mayor de mujeres no tiene ningún hijo y las que tienen hijos, cada vez tienen menos. La edad de maternidad cada vez se retrasa más, lo que está provocando a su vez que la edad normativa entre las mujeres sea más alta. Esta caída de la fecundidad, también se debe a factores relacionados directamente con lo económico-laboral, que hace que los recursos necesarios para la crianza de un hijo sean insuficientes o que el mercado laboral impida la compatibilidad de la vida profesional con la vida familiar. Esto también es una señal de la escasa presencia de un marco institucional que apoye a la maternidad con políticas públicas que fomenten y faciliten a las mujeres el poder ser madres sin renunciar a una carrera profesional exitosa. 


\section{REFERENCIAS BIBLIOGRÁFICAS}

Becker, G. S., \& de Grado, C. P. (1987). Tratado sobre la familia (Vol. 1). Madrid: Alianza Editorial.

Bernardi, F., \& Requena, M. (2004). La caída de la fecundidad y el déficit de natalidad en España. Revista Española de Sociología, 3, 29-49.

Castro Martín, T., \& Rosero-Bixby, L. (2011). Maternidades y fronteras. La fecundidad de las mujeres inmigrantes en España. Revista Internacional de Sociología, 69(M1), 105138. https://doi.org/10.3989/ris.2011.im1.388

Castro, T., \& Seiz, M. (2014). La transformación de las familias en España desde una perspectiva socio-demográfica. VII Informe Sobre Exclusión y Desarrollo Social En España, 1-35.

Delgado, M., Zamora López, F., \& Barrios, L. (n.d.). Déficit de fecundidad en España: factores demográficos que operan sobre una tasa muy inferior al nivel de reemplazo. Delgado Margarita; Barrios, Laura; Zamora, Francisco (REIS n. 115. ARTÍCULOS). REIS. Revista Española de Investigaciones Sociológicas, 197-222.

Esping-Andersen, G., Arpino, B., Baizán, P., Bellani, D., Castro-Martín, T., Creighton Mathew J., ... Rutigliano, R. (2013). El déficit de natalidad en Europa. Colección Estudios Sociales, (36). Retrieved from http://backend.demografia.ieg.csic.es/upload/files/ cv/pdf/publication/a6139b8ce764004722637a4d3e1c5e5e.pdf

Esteve, A., Devolder, D., \& Domingo, A. (2016). La infecunidad en España: tic-tac, tic-tac!!, 1-4.

Hakim, C. (1998). Developing a Sociology for the Twenty-First Century: Preference Theory. The British Journal of Sociology, 49(1), 137. doi:10.2307/591267

Hakim, C. (2000). Work-lifestyle choices in the 21st century. Oxford, England: Oxford University Press

Kohler, H.-P., Billari, F. C., \& Ortega, J. A. (2004). The Emergence of Lowest-Low Fertility in Europe During the 1990s. Population and Development Review, 28(4), 641-680.

Lesthaeghe, R. (1995). The second demographic transition in Western countries: An interpretation. Gender and family change in industrialized countries, 17-62.

López-Roldán, P., \& Fachelli, S. (2016). Análisis de regresión logística. Metodología de la investigación social cuantitativa. 
Martín Patino, J. M. (2017). Informe España 2018 (Vol. 91).

Miguel, J. M. de. (1980). Sociología de la población y control de la natalidad en españa. Reis, (10), 15. https://doi.org/10.2307/40182773

Mínguez, A. M. (2012). Familia, empleo femenino y reproducción en España: Incidencia de los factores estructurales. Papers, 97(2), 461-495.

Reher, D., \& Requena, M. (2018). Childlessness in Twentieth-Century Spain: A Cohort Analysis for Women Born 1920-1969. European Journal of Population, 35(1), 1-28. https://doi.org/10.1007/s10680-018-9471-7

Sampedro, R., Gómez, M. V., \& Montero, M. (2002). Maternidad tardía: Incidencia, perfiles y discrusos. Empiria: Revista de Metodología En Ciencias Sociales, 27.

Van de Kaa, D. J. (1987). Europe's second demographic transition. Population bulletin, 42(1), 1-59.

Vidal-Coso, E., \& Miret-Gamundi, P. (2017). Características de las madres primerizas y de los padres primerizos en la España del siglo xxI / Characteristics of First-time Parents in Spain along the 21st Century. Revista Española de Investigaciones Sociológicas, 115-138. https://doi.org/10.5477/cis/reis.160.115 